\title{
Radiation Therapy as Definitive Local Treatment in Patients with Limited-Stage Small Cell Carcinoma of the Bladder: Does total dose matter?
}

\author{
Elizabeth Germino, Benjamin W. Fischer-Valuck, Soumon Rudra, Yuan James Rao, Jessika \\ Contreras, Christopher Abraham, Jeff Michalski and Hiram Gay* \\ Department of Radiation Oncology, Washington University School of Medicine, St. Louis, MO, USA
}

\begin{abstract}
.
Purpose: To determine whether total radiation dose affects survival outcomes for patients with small cell carcinoma of the bladder (SCCB).

Methods: We queried the National Cancer Database (NCDB) for patients with limited stage SCCB undergoing multimodality treatment and retrospectively analyzed survival outcomes according to total radiation dose received.

Results: Patients aged 41-79 receiving a total radiation dose of 54 Gy or greater had a significant improvement in overall survival compared to those receiving less than $54 \mathrm{~Gy}$, with a median overall survival of 58.9 months ( $95 \%$ confidence interval [CI] 37.2-80.6 months) compared to 21.5 months (95\% CI $15.2-27.8$ months) $(p<0.05)$. There was no difference in outcomes for patients receiving between 54 and $60 \mathrm{~Gy}$ compared to those receiving $60 \mathrm{~Gy}$ or higher. There was also no difference in outcomes based on total radiation dose for patients 80 years and older.

Conclusions: For patients aged 79 or younger with limited stage SCCB, total radiation dose of 54 Gy or greater is associated with better overall survival.
\end{abstract}

Keywords: Small cell carcinoma of the bladder, NCDB, bladder cancer, national cancer database, radiation therapy

\section{INTRODUCTION}

Small cell carcinoma of the bladder (SCCB) is a rare and aggressive disease estimated to comprise $<1 \%$ of all urinary bladder cancers [1-7]. It is most commonly diagnosed in white, elderly males $[2-6,8,9]$ and has a significantly worse prognosis compared to urothelial bladder cancer. A recent

${ }^{*}$ Correspondence to: Hiram Gay, MD, Department of Radiation Oncology, Washington University, St. Louis, MO, 4921 Parkview Place St. Louis, MO 63110, USA. Tel.: +1 314362 8516; E-mail: hgay@radonc.wustl.edu. population-based analysis using the Surveillance, Epidemiology, and End Results (SEER) database reported a median overall survival of 12 months for all patients [10]. Given the infrequency of SCCB and lack of prospective clinical trials, most evidence for treatment strategies has been drawn from retrospective studies and extrapolations from treatment of small cell carcinoma in other disease sites. While radical cystectomy is considered to be definitive local therapy for SCCB, recent studies have shown that similar to treatment of small cell carcinoma of the lung, systemic chemotherapy, particularly in the 
neoadjuvant setting, can improve survival compared to local therapy alone $[11,12]$. Long-term survivors have also been described with a bladder-conservation approach combining chemotherapy with definitive local radiation treatment [13-17]. It is difficult to compare these strategies head-to-head without a prospective study, but either cystectomy or radiotherapy are recommended as definitive local treatment according to the current National Cancer Care Network (NCCN) guidelines for bladder cancers with the much less common small cell histology [18].

While most retrospective studies that have utilized definitive radiotherapy report total doses between 56 and 70 Gy $[6,13-17]$, there are have been no published studies that specifically address the optimal dose of radiation for these patients. Furthermore, small sample sizes have limited the ability to compare outcomes based on radiation dose. In this study, we used the National Cancer Database (NCDB) to analyze a large cohort of patients who received chemoradiation for SCCB, with the goal to evaluate whether total radiation dose correlated with overall survival.

\section{MATERIALS/METHODS}

\section{Data source and study population}

The NCDB Participant User File was queried for de-identified data from 2004 and 2013 for patients 18-90 years of age diagnosed with small cell carcinoma of the bladder [International Classification of Disease for Oncology, 3rd edition (ICD-0-3) codes 8002,8041 , and 8043] The NCDB is a joint program of the American College of Surgeons and the American Cancer Society. Data from approximately $70 \%$ of patients diagnosed at Commission on Cancer accredited cancer centers is included and includes patient, tumor, and treatment characteristics. Data elements are collected and submitted to the NCDB from commission-accredited oncology registries using standardized coding and data item definitions, including some details not available from SEER, such as radiotherapy dose/technique, chemotherapy use/timing, and comorbidity. The Participant User File contains de-identified patient and center information and was exempt from Institutional Review Board review.

We limited our analysis to patients with muscle invasive disease and clinically negative lymph nodes (cT1-4 N0) who received radiation therapy to the bladder or pelvis (Radiation Treatment Volume codes
29 and 34) in conjunction with chemotherapy (including both single and multi-agent therapy). Patients with metastatic disease and incomplete treatment data were excluded. All patients underwent transurethral resection of bladder tumor (TURBT) for pathologic diagnosis and prior to definitive treatment. Total radiation dose received was defined as the sum of the regional dose delivered plus any boost dose. Fourteen cases were excluded because the total dose calculated was less than $40 \mathrm{~Gy}$ or greater than 80 Gy which likely represented either palliative treatment or potential coding errors.

\section{Statistical analysis}

The Pearson chi square test was used to compare baseline characteristics of patients receiving either a higher (greater than or equal to 54 Gy) or lower (less than $54 \mathrm{~Gy}$ ) total radiation dose. Overall survival (OS) was estimated using the Kaplan-Meier method, calculated from diagnosis until death, censoring at last follow-up for patients who were alive. The log-rank test was used to determine whether survival curves were statistically different, defined as a $p$ value $<0.05$. SPSS Statistics v. 24 (IBM Corporation; Armonk, New York) was used for all statistical analyses.

\section{RESULTS}

One hundred and eighty patients were identified with SCCB that received chemoradiation and met the inclusion criteria described above. Median follow-up was 24.8 months (range, 3.7 to 131.6 months). Consistent with previously reported demographic data, patients tended to be older, male, and white. Forty-four $(24.4 \%)$ patients received a total radiation dose of less than $54 \mathrm{~Gy}$ (low-dose), and $136(75.6 \%)$ patients received a total dose of greater than or equal to $54 \mathrm{~Gy}$ (high-dose), with a range from 40.0 to $77.4 \mathrm{~Gy}$. Most patients received a total dose between 45 and $64.8 \mathrm{~Gy}$, with the most common regional dose (i.e. before boost) being 45 Gy (Fig. 1). A summary of baseline characteristics for patients receiving low-dose versus high-dose radiation is provided in Table 1 . There was no statistically significant difference between the two groups for any of the characteristics analyzed, with the exception of clinical $\mathrm{T}$ stage; more patients who received high-dose were assigned a stage of T1 $(24.3 \%$ compared to $6.8 \%$ for the lower dose treatment group). However, even when these cases 

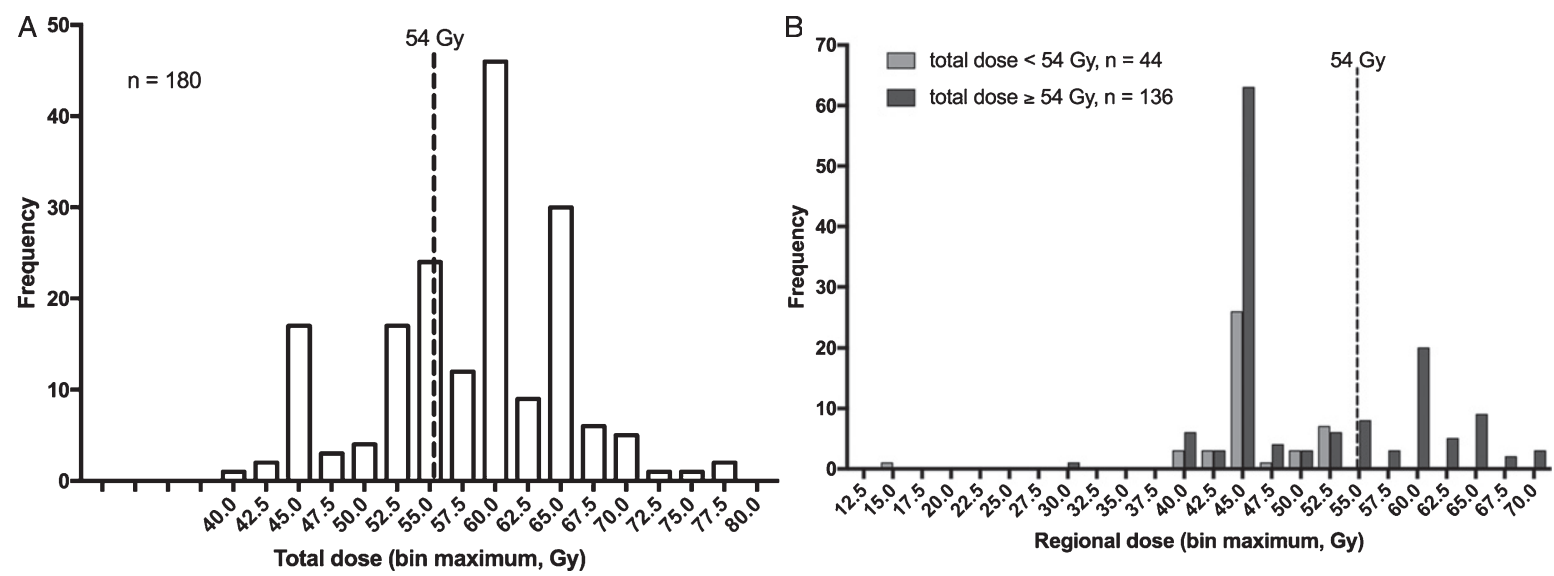

Fig. 1. Distribution of delivered radiation doses. A. Histogram depicting the frequency of total radiation dose delivered. Most patients received a total dose between 45 and $64.8 \mathrm{~Gy}$ (range 40-77.4 Gy). B. Grouped histogram of regional dose delivered (before boost), segregated by total dose received.

Table 1

Baseline characteristics for patients receiving $<54$ Gy versus $\geq 54$ Gy total radiation dose

\begin{tabular}{|c|c|c|c|c|c|}
\hline \multirow[t]{2}{*}{$\begin{array}{l}\text { Total radiation } \\
\text { dose }\end{array}$} & \multicolumn{2}{|c|}{$\begin{array}{c}<54 \\
\text { Gy }\end{array}$} & \multicolumn{2}{|c|}{$\begin{array}{c}\geq 54 \\
\text { Gy }\end{array}$} & \multirow[t]{2}{*}{$\begin{array}{c}p \\
\text { value }\end{array}$} \\
\hline & number & percent & number & percent & \\
\hline \multicolumn{6}{|l|}{ Age } \\
\hline$\geq 80$ & 15 & 34.1 & 41 & 30.1 & \multirow[t]{2}{*}{0.623} \\
\hline $41-79$ & 29 & 65.9 & 95 & 69.9 & \\
\hline \multicolumn{6}{|l|}{ Sex } \\
\hline Male & 35 & 79.5 & 101 & 74.3 & \multirow[t]{2}{*}{0.479} \\
\hline Female & 9 & 20.5 & 35 & 25.7 & \\
\hline \multicolumn{6}{|l|}{ Race } \\
\hline White & 43 & 97.7 & 126 & 92.6 & \multirow[t]{2}{*}{0.221} \\
\hline Other & 1 & 2.3 & 10 & 7.4 & \\
\hline \multicolumn{6}{|l|}{ Facility Type } \\
\hline Academic & 13 & 29.5 & 45 & 33.1 & \multirow[t]{2}{*}{0.662} \\
\hline Other & 31 & 70.5 & 91 & 66.9 & \\
\hline \multicolumn{6}{|l|}{ Insurance status } \\
\hline Not Insured & 2 & 3.6 & 0 & 0.0 & \multirow[t]{6}{*}{0.09} \\
\hline Private Insurance/Managed Care & 13 & 23.2 & 31 & 22.8 & \\
\hline Medicaid & 1 & 1.8 & 7 & 5.1 & \\
\hline Medicare & 36 & 64.3 & 93 & 68.4 & \\
\hline Other Government & 4 & 7.1 & 3 & 2.2 & \\
\hline Insurance Status Unknown & 0 & 0.0 & 2 & 1.5 & \\
\hline \multicolumn{6}{|l|}{ Charlson/Deyo Score } \\
\hline 0 & 31 & 70.5 & 93 & 68.4 & \multirow[t]{3}{*}{0.633} \\
\hline 1 & 9 & 20.5 & 35 & 25.7 & \\
\hline 2 & 4 & 9.1 & 8 & 5.9 & \\
\hline \multicolumn{6}{|l|}{ AJCC Clinical T } \\
\hline 1 & 3 & 6.8 & 33 & 24.3 & \multirow[t]{4}{*}{0.028} \\
\hline 2 & 31 & 70.5 & 89 & 65.4 & \\
\hline 3 & 6 & 13.6 & 8 & 5.9 & \\
\hline 4 & 4 & 9.1 & 6 & 4.4 & \\
\hline \multicolumn{6}{|l|}{ Year Diagnosed } \\
\hline 2004-2009 & 25 & 56.8 & 78 & 57.4 & \multirow[t]{2}{*}{0.95} \\
\hline 2010-2013 & 19 & 43.2 & 58 & 42.6 & \\
\hline
\end{tabular}

were excluded, similar trends held in subsequent analyses.

The median OS for the entire cohort was 34.8 months (95\% confidence interval [CI], 19.3 to 50.3 months), with estimated 3- and 5-year OS rates of $50 \%$ and $38 \%$, respectively (Fig. 2). Median OS for patients treated to $54 \mathrm{~Gy}$ or higher was 42.8 months (95\% CI 19.0 to 66.6 months), while the median 


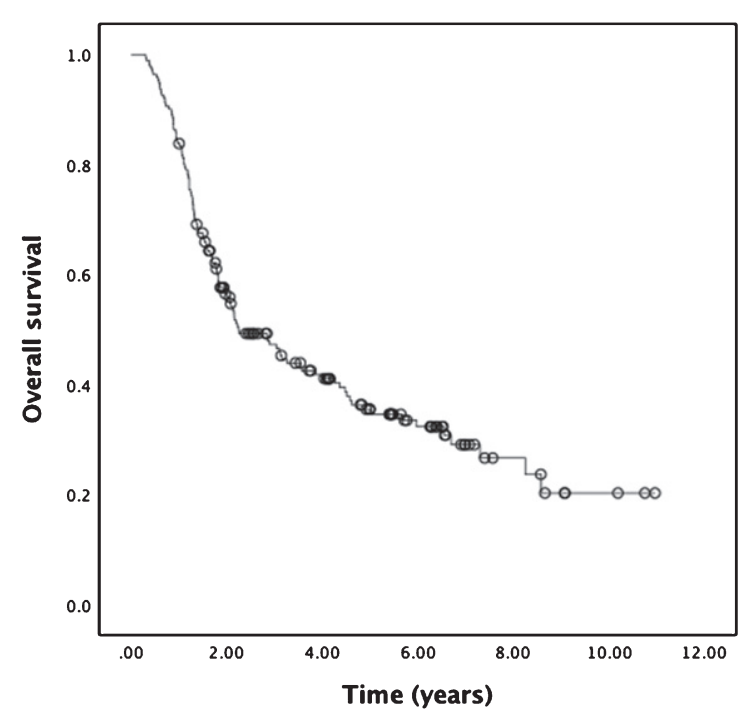

Fig. 2. Kaplan-Meier analysis for OS of entire cohort. Median OS was 34.8 months $(n=180)$, with estimated 3- and 5-year OS rates of $50 \%$ and $38 \%$, respectively.

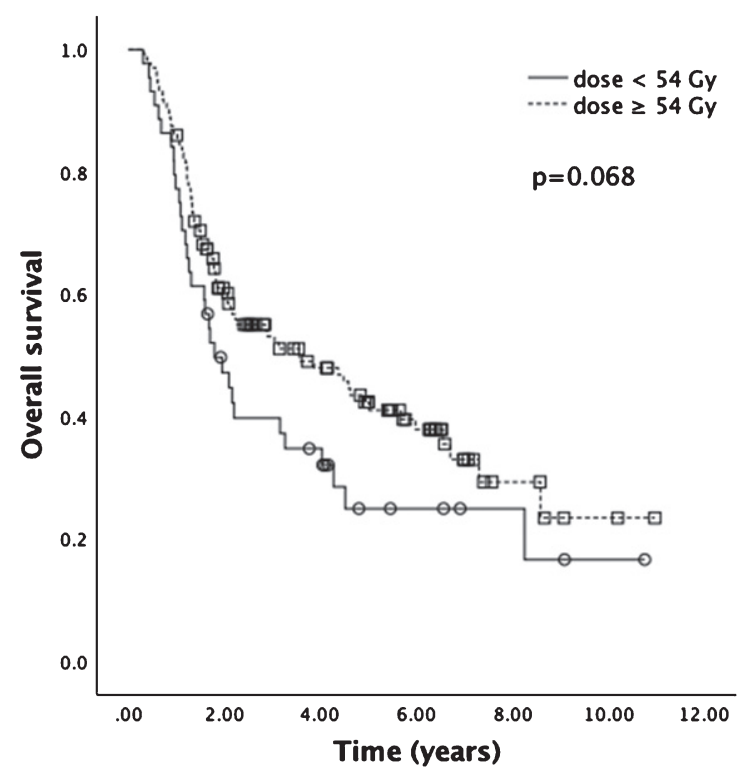

Fig. 3. Kaplan-Meier analysis for OS of patients receiving total radiation dose $<54 \mathrm{~Gy}$ or $\geq 54 \mathrm{~Gy}$. Median OS for patients receiving $\geq 54 \mathrm{~Gy}$ was 42.8 months, compared to 21.5 months for patients receiving $<54$ Gy $(p=0.068)$.

OS for patients receiving less than $54 \mathrm{~Gy}$ was 21.5 months (95\% CI 16.5 to 27.7 months) (Fig. 3). The difference in median survival for these two treatment groups trended toward statistical significance $(p=0.068)$.
Stratifying total radiation dose by age group, median OS was significantly increased with a higher radiation dose for patients 41-79 years old (median OS 58.9 months, 95\% CI 37.2-80.6 months, compared to median OS for patients receiving a lower dose of 21.5 months, 95\% CI 15.2-27.8 months, $p=0.019)($ Fig. 4A), but the difference was not statistically significant for patients 80 and older $(p=0.819)$ (Fig. 4B). Notably, in patients aged $41-79$ receiving $60 \mathrm{~Gy}$ or higher, there was no difference in survival compared to patients receiving between 54 and $60 \mathrm{~Gy}$ $(p=0.291)$ (Fig. 4C). When age groups were further segregated by comorbidity score, a trend for improvement in OS with higher radiation dose was still observed for younger patients with no comorbidities (median OS 58.9, 95\% CI 23.0-94.8 months; $p=0.051)$ (Supplemental Figure 1).

\section{DISCUSSION}

The low incidence of small cell bladder cancer has precluded randomized controlled trials to establish optimal treatment strategies. One prospective trial [15] and a number of retrospective studies [6, 13, $14,16,17]$ have suggested that reasonable outcomes can be achieved with a bladder-conservation strategy of chemotherapy plus definitive dose local radiation for patients with limited-stage disease. However, there have been no published studies that specifically address the optimal dose of radiation.

Here, we report an analysis of NCDB data directly comparing survival of patients with limited stage SCCB undergoing multimodality treatment, according to radiation dose received. There are several advantages to using this cohort; first, it includes a relatively large number of patients spanning multiple treatment centers and includes a modern era of treatment. The patients included in the analysis tended to be white males, with a mean age of 72.1 years, in agreement with previously published epidemiologic data $[2-6,8,9]$. Prognosis of patients undergoing chemoradiation was also comparable with an overall median survival for the entire cohort of 34.8 months and an estimated 5-year overall survival rate of $38.0 \%$. Reported median survival in the literature for limited-stage SCCB with treatment ranges from 7-159 months [11, 12, 19], while studies specifically considering bladder-preservation therapy have described a median survival of $15-41$ months $[6,14$, $15,17]$. 

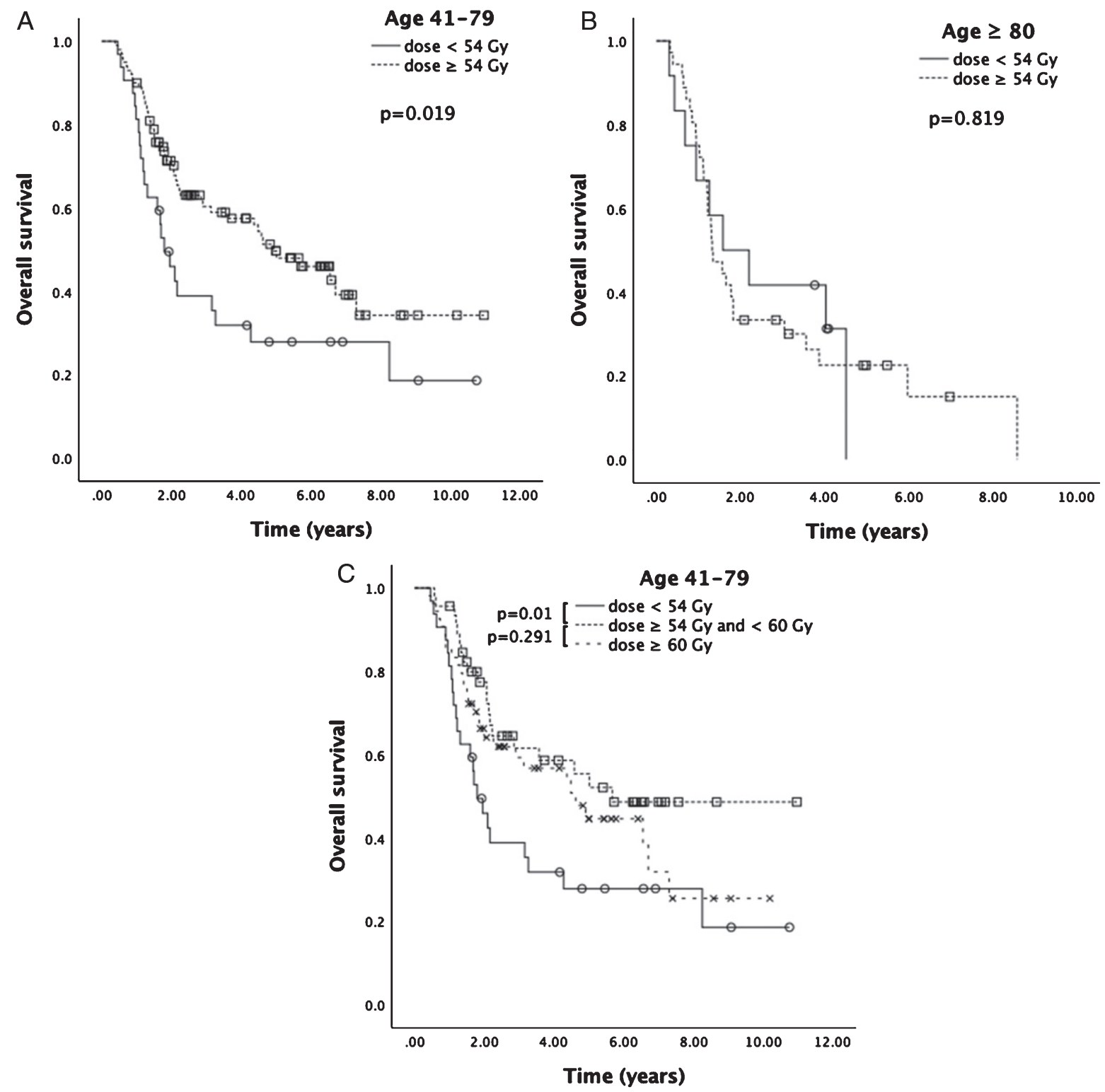

Fig. 4. Kaplan-Meier analysis for OS stratified by age. A. Median OS for patients age 41-79 receiving $\geq 54$ Gy was 58.9 months; median OS was 21.5 months for patients receiving $<54 \mathrm{~Gy}(p=0.019)$. B. There was no statistically significant difference in median OS for patients 80 and older receiving $\geq 54$ Gy compared to $<54$ Gy ( 15.9 months versus 19.0 months, respectively, $p=0.819)$. C. For patients age $41-79$, there was no statistically significant difference in median OS for total dose $\geq 60 \mathrm{~Gy}(55.4$ months, $n=54$ ) compared to total dose $\geq 54 \mathrm{~Gy}$ and <60 Gy (68.1 months, $n=46 ; p=0.291$ ).

Analysis of our cohort of patients receiving radiation therapy in conjunction with systemic chemotherapy demonstrated a statistically significant benefit of higher total doses for patients younger than 79 years, with a median OS of 58.9 months (versus 21.5 months for those receiving a lower total dose). While total dose of 54 Gy or greater was beneficial in this group, patients who received $60 \mathrm{~Gy}$ or greater did not gain any additional improvement in overall survival, but given the small number of patients it is unlikely that the data is powered to detect a change such as this. This is notable since a review of the literature indicates that it is not uncommon for patients to receive total doses greater than $60 \mathrm{~Gy}$ and as much as $70 \mathrm{~Gy}$ [13-17]. Our analysis suggests that similar outcomes might be achievable with a lower dose and therefore possibly allow for less acute and long-term toxicities. 
Age greater than 79 years carried a worse prognosis in general, and for these patients, total radiation dose did not significantly impact overall survival, regardless of comorbidity score. However, it is likely that this group of patients included many who were poor surgical candidates, and it is difficult to conclusively say whether lower dose radiation therapy could replace radical cystectomy for elderly patients otherwise qualified to have definitive surgical treatment. Elderly patients may have also received less aggressive chemotherapy regimens or had other factors carrying a worse prognosis not captured in our database. Still, these data suggest that doses lower than 54 Gy might be reasonable in order to avoid unnecessary toxicity in elderly patients with SCCB whose best option is chemoradiation.

An important consideration when analyzing retrospective data is selection bias; younger, healthier patients tend to be treated more aggressively, and indeed we found that the presence of comorbidities significantly impacted survival in our multivariate analysis. However, we still observed a significant effect of radiation dose when limiting analysis to younger patients with no comorbidities. While selection bias cannot be completely eliminated in a retrospective analysis such as this one, these data suggest that radiation dose may indeed be an independent predictor of survival for younger patients undergoing bladder-preservation with chemoradiation therapy.

While this is still one of the larger analyses performed to date, our study is limited by constraints of database queries. Specifically, we do not have data for chemotherapy regimens and dosing, which is a potential confounder. We also were unable to address fractionation of radiation dose in this analysis. Regardless of dose, patients were most likely to receive either photon therapy or IMRT compared to conformal/3D-RT. The percentage of patients in each group receiving these different treatment modalities was similar and therefore unlikely to skew our results based on toxicities inherent to treatment modality. Notably, we only considered the primary endpoint of overall survival, and not disease-related mortality; we were also unable to take into account the influence of radiation dose on factors such as adverse events and quality of life. Lastly, when we searched the database, we captured all of the included patients which met eligibility criteria for combined chemoradiation therapy. We specifically did not exclude surgery following chemoradiation from our criteria because we wanted to capture outcomes of all patients treated with definitive chemoradiation including those who may have failed and gone on to salvage surgery. Two patients in our cohort underwent radical cystectomy after definitive intent chemoradiation.

Our results suggest that further investigation of outcomes based on radiation dose for SCCB would be of utility. Based on our analysis, for patients age 79 or under receiving chemoradiation as part of a bladderconservation approach, we recommend considering a total radiation dose between 54 and $60 \mathrm{~Gy}$. For patients older than 79 , our analysis did not correlate higher doses with improved overall survival, possibly due to other comorbidities inherent to elderly patients not captured in our database. Given the rarity of this disease we feel that the literature and our own analysis continue to support an individualized approach to treatment, which optimally would include either neoadjuvant or induction chemotherapy followed by definitive local therapy with either surgery or chemoradiation.

\section{FUNDING SOURCES}

None.

\section{SUPPLEMENTARY MATERIAL}

The supplementary material is available in the electronic version of this article: http://dx.doi.org/10. 3233/BLC-180165.

\section{CONFLICT OF INTEREST}

Authors have no conflict of interests.

\section{REFERENCES}

[1] Abrahams NA, Moran C, Reyes AO, Siefker-Radtke A, Ayala AG. Small cell carcinoma of the bladder: A contemporary clinicopathological study of 51 cases. Histopathology. 2005;46:57-63.

[2] Cheng L, Pan CX, Yang XJ, Lopez-Beltran A, MacLennan GT, Lin H, et al. Small cell carcinoma of the urinary bladder: A clinicopathologic analysis of 64 patients. Cancer. 2004;101:957-62.

[3] Choong NW, Quevedo JF, Kaur JS. Small cell carcinoma of the urinary bladder. The Mayo Clinic experience. Cancer. 2005;103:1172-8.

[4] Ismaili N, Elkarak F, Heudel PE, Flechon A, Droz JP. Small cell cancer of the bladder: The Leon-Berard cancer centre experience. Indian J Urol. 2008;24:494-7.

[5] Koay EJ, Teh BS, Paulino AC, Butler EB. A Surveillance, Epidemiology, and End Results analysis of small cell carcinoma of the bladder: Epidemiology, prognostic variables, and treatment trends. Cancer. 2011;117:5325-33. 
[6] Lohrisch C, Murray N, Pickles T, Sullivan L. Small cell carcinoma of the bladder: Long term outcome with integrated chemoradiation. Cancer. 1999;86:2346-52.

[7] Quek ML, Nichols PW, Yamzon J, Daneshmand S, Miranda G, Cai J, et al. Radical cystectomy for primary neuroendocrine tumors of the bladder: The university of southern california experience. J Urol. 2005;174:93-6.

[8] Mangar SA, Logue JP, Shanks JH, Cooper RA, Cowan RA, Wylie JP. Small-cell carcinoma of the urinary bladder: 10-year experience. Clin Oncol (R Coll Radiol). 2004;16:523-7.

[9] Siefker-Radtke AO, Dinney CP, Abrahams NA, Moran C, Shen Y, Pisters LL, et al. Evidence supporting preoperative chemotherapy for small cell carcinoma of the bladder: A retrospective review of the M. D. Anderson Cancer Experience. J Urol. 2004;172:481-4.

[10] Schreiber D, Rineer J, Weiss J, Leaf A, Karanikolas N, Rotman M, et al. Characterization and outcomes of small cell carcinoma of the bladder using the surveillance, epidemiology, and end results database. Am J Clin Oncol. 2013;36:126-31.

[11] Lynch SP, Shen Y, Kamat A, Grossman HB, Shah JB, Millikan RE, et al. Neoadjuvant chemotherapy in small cell urothelial cancer improves pathologic downstaging and long-term outcomes: Results from a retrospective study at the MD Anderson Cancer Center. Eur Urol. 2013;64:307-13.

[12] Siefker-Radtke AO, Kamat AM, Grossman HB, Williams DL, Qiao W, Thall PF, et al. Phase II clinical trial of neoadjuvant alternating doublet chemotherapy with ifosfamide/doxorubicin and etoposide/cisplatin in smallcell urothelial cancer. J Clin Oncol. 2009;27:2592-7.

[13] Asmis TR, Reaume MN, Dahrouge S, Malone S. Genitourinary small cell carcinoma: A retrospective review of treatment and survival patterns at The Ottawa Hospital Regional Cancer Center. BJU Int. 2006;97:711-5.

[14] Bex A, de Vries R, Pos F, Kerst M, Horenblas S. Longterm survival after sequential chemoradiation for limited disease small cell carcinoma of the bladder. World J Urol. 2009;27:101-6.

[15] Bex A, Nieuwenhuijzen JA, Kerst M, Pos F, van Boven $\mathrm{H}$, Meinhardt $\mathrm{W}$, et al. Small cell carcinoma of bladder: A single-center prospective study of 25 cases treated in analogy to small cell lung cancer. Urology. 2005;65: 295-9.

[16] Bryant CM, Dang LH, Stechmiller BK, Gilbert SM, Morris CG, Zlotecki RA. Treatment of small cell carcinoma of the bladder with chemotherapy and radiation after transurethral resection of a bladder tumor. Am J Clin Oncol. 2016;39:69-75.

[17] Meijer RP, Meinhardt W, van der Poel HG, van Rhijn BW, Kerst JM, Pos FJ, et al. Local control rate and prognosis after sequential chemoradiation for small cell carcinoma of the bladder. Int J Urol. 2013;20:778-84.

[18] National Comprehensive Cancer Network. Bladder Cancer (Version 5.2017). https://www.nccn.org/professionals/ physician_gls/pdf/bladder.pdf. Retrieved June 23, 2017.

[19] Holmang S, Borghede G, Johansson SL. Primary small cell carcinoma of the bladder: A report of 25 cases. J Urol. 1995;153:1820-2. 